\title{
Dilatation of the pulmonary autograft and native aorta after the Ross procedure: A comprehensive echocardiographic study
}

\author{
Obaid Aljassim, MD, ${ }^{\mathrm{a}, \mathrm{d}}$ Gunnar Svensson, MD, PhD,${ }^{\mathrm{a}, \mathrm{d}}$ Sossio Perrotta, MD, ${ }^{\mathrm{a}}$ Anders Jeppsson, MD, PhD, ${ }^{\mathrm{a}, \mathrm{d}}$ \\ and Odd Bech-Hanssen, MD, $\mathrm{PhD}^{\mathrm{b}, \mathrm{c}, \mathrm{d}}$
}

\begin{abstract}
Objectives: Dilatation of the pulmonary autograft has been observed after the Ross procedure. Whether the remaining native aorta dilates is not known. The aim of the study was to describe the prevalence and severity of autograft and native aortic dilatation over time and to identify possible determinants.
\end{abstract}

\begin{abstract}
Methods: Ninety-one adult patients underwent the Ross procedure with the full root replacement technique. In $31(34 \%)$ patients, the ascending aorta was downsized during surgical intervention. A baseline postoperative echocardiographic investigation was performed. A comprehensive investigation of the aorta from the annulus to the proximal descending aorta was performed $(\mathrm{n}=71)$ after a median follow-up of 8.9 years. An intermediate investigation was performed $(\mathrm{n}=29)$ after a median of 7.6 years. Autograft and native aortic dimensions were compared over time and with those of a control group $(n=38)$. For each patient in the study group, the expected aortic dimensions were predicted based on findings in the control group. Enlargement was defined as a $z$ score of greater than 1.96 from the predicted value.
\end{abstract}

Results: The autograft and native aortic dimensions increased significantly from baseline to the intermediate follow-up and continued to increase to the final follow-up. The proportion of patients with enlarged autografts and proximal ascending aortas was $13 \%$ and $16 \%$ at baseline, increasing to $33 \%(P=.006)$ and $44 \%$ $(P=.0014)$, respectively, at the end of follow-up. Enlargement of the aorta at the final follow-up was related to larger baseline pulmonary autograft dimensions but not to native bicuspid valve or the need to downsize the aortic root.

Conclusions: Pulmonary autograft dilatation is common after the Ross procedure in adults. The dilatation progresses over time and is often accompanied by dilatation of the native aorta. (J Thorac Cardiovasc Surg 2011;142:634-40)

Supplemental material is available online.

The Ross procedure is an alternative in aortic valve replacement for adults. ${ }^{1-6}$ The complexity of the operation consists of the need to replace both the aortic and pulmonary valves and implantation of coronary arteries. The risk for reoperation remains an important limitation. Originally the Ross procedure was described as a subcoronary implant. ${ }^{7}$ The full root technique was introduced in $1989^{6}$ and had the advantage of a technically easier way of providing the patient with a competent valve without an outflow

From the Departments of Cardiovascular Surgery and Anesthesia, ${ }^{a}$ Clinical Physiology, ${ }^{\mathrm{b}}$ and Cardiology, ${ }^{\mathrm{c}}$ Sahlgrenska University Hospital, and the Department of Molecular and Clinical Medicine, ${ }^{\mathrm{d}}$ Institute of Medicine, Sahlgrenska Academy, University of Gothenburg, Gothenburg, Sweden.

Disclosures: Authors have nothing to disclose with regard to commercial support.

Received for publication July 15, 2010; revisions received Oct 22, 2010; accepted for publication Nov 12, 2010; available ahead of print Jan 31, 2011.

Address for reprints: Odd Bech-Hanssen, MD, PhD, Department of Cardiology, Sahlgrenska University Hospital, SE-413 45 Göteborg, Sweden (E-mail: odd. bech-hanssen@klinfys.gu.se).

0022-5223/\$36.00

Copyright (C) 2011 by The American Association for Thoracic Surgery doi: $10.1016 /$ j.jtcvs.2010.11.025 gradient. However, several reports indicate that progressive pulmonary autograft dilatation with or without regurgitation can occur after the full root replacement, and this might be a cause of reoperation. ${ }^{2,7}$

In our series, routine follow-up echocardiographic investigations occasionally showed a dilated autograft but in some cases also showed dilatation of the native aorta. This finding prompted us to perform a comprehensive transthoracic investigation of the aorta from the annulus to the proximal part of the descending aorta, including the aortic arch. The aims of this study were to evaluate the prevalence and severity of the pulmonary autograft and native aortic dilatation after the Ross procedure in adults, to study the progression of dilatation over time, and to identify possible determinants.

\section{MATERIAL AND METHODS Patients}

From January 1995 to January 2002, a total of 91 adult patients underwent the Ross procedure at Sahlgrenska University Hospital by using the full root replacement technique. The mean age was $45 \pm 12$ years (range, 17-66 years). Fifteen patients had a previous median sternotomy. Four patients had endocarditis: 1 had an aortic mechanical valve with endocarditis and paravalvular leakage, 1 had endocarditis after a previous commisurotomy, and 2 had native valve endocarditis. Patients' demographics and preoperative characteristics are shown in Table 1. 
The patients underwent an echocardiographic investigation within the first postoperative week (baseline investigation, $n=63$ ). These baseline investigations were performed by different investigators, and the distal part of the ascending aorta or the aortic arch was not regularly examined. In $71(78 \%)$ patients, a more comprehensive echocardiographic follow-up investigation was performed 8.9 years (range, 2.2-14.1 years) after the initial procedure. Eight patients were not alive at the time of echocardiographic follow-up, and $12(14 \%)$ were unavailable for investigation. In 29 patients, an intermediate investigation with the same comprehensive protocol as at the end of follow-up was performed 7.6 years (range, 3.8-10 years) postoperatively. The intermediate and end follow-up investigations were carried out by a single investigator. Thirty-eight healthy adults were investigated by using the same protocol and by the same investigator and served as a control group. The control subjects $(60 \%$ women; mean \pm standard deviation age, $51 \pm 9$ years; and body surface area, $1.83 \pm 0.16 \mathrm{~m}^{2}$ ) had no history of heart disease, hypertension, or diabetes mellitus.

Patients' data were collected retrospectively from medical records and from prospective echocardiographic investigations. Follow-up was $100 \%$ complete regarding mortality and reoperation. The regional ethical research committee waived patient consent for the study.

\section{Operative Procedure}

All patients had a preoperative transthoracic echocardiographic or transesophageal echocardiographic investigation. Pulmonary autograft replacement of the aortic valve was used only in patients with no or trivial pulmonary valve regurgitation in the preoperative evaluation. The surgical technique used was full freestanding aortic root replacement with a pulmonary autograft in all cases. The right ventricular outflow tract was reconstructed with a fresh or cryopreserved homograft from our own homograft bank that was collected from cardiac transplant recipients or donor hearts. The operative technique has been published in detail previously. ${ }^{8}$ In the first 24 cases, the left coronary artery ostium was retained with a tongue of the aortic wall, and the proximal autograft anastomosis was performed with a continuous suture line. The diameter of the pulmonary roots was not measured, and cases with a dilated annulus, aorta, or both were excluded by visual inspection.

In the latest 67 cases, the surgical technique was changed. The left ostium was reimplanted in the autograft, and a proximal anastomosis was performed with interrupted suture lines. In cases with diameter mismatch between the pulmonary and aortic root measured with intraoperative transesophageal echocardiographic analysis, the aortic root (annulus, proximal ascending aorta, or both) was adjusted to the size of the pulmonary root.

A reduction of the aortic annulus was performed in cases with moderate dilatation $(<5 \mathrm{~mm}$ mismatch) with a strip of Teflon (PTFE-felt; Meadox Medical, Inc, Oakland, Calif) in the area between the 2 fibrous trigones where dilatation occurred. In cases of severe dilatation ( $\geq 5$ - $\mathrm{mm}$ mismatch), the Ross procedure was not performed. The aorta was reduced with an aortoplasty if the diameter was larger than the diameter of the distal end of the pulmonary autograft. The aortic wall was duplicated, sutured, and reinforced with a Teflon strip at each side. In 1 case of an aneurysm of the aorta, the ascending aorta was removed, and an interposition graft was used.

\section{Transthoracic Echocardiographic Investigation}

The transthoracic investigation of the left ventricular outflow tract, aortic valve, and aorta was performed with the patient in the standard left lateral position but also in the right lateral position with the transducer at the right parasternal border. With the transducer at this site, it is possible to visualize the middle and distal part of the ascending aorta (see Figure E1). This measurement was especially important in the patients undergoing the Ross procedure in which the measurement in the middle or distal part represents the native aorta, whereas the measurement in the standard parasternal long-axis projection might be either the autograft or the native aorta. With the patient in the supine position with the transducer in the suprasternal notch, we investigated the aortic arch and the distal part of the ascending aorta (see Figure E1). The 2-dimensional data were stored
TABLE 1. Demographics and preoperative characteristics

\begin{tabular}{lc}
\hline No. of patients & 91 \\
Mean age (y) & $45 \pm 12$ \\
Female sex & $30(33 \%)$ \\
NYHA & \\
Class I & $31(34 \%)$ \\
Class II & $40(44 \%)$ \\
Class III & $16(18 \%)$ \\
Class IV & $4(4 \%)$ \\
Previous median sternotomy & $15(16 \%)$ \\
Previous valve commisurotomy & $10(11 \%)$ \\
Endocarditis & $4(4 \%)$ \\
Hypertension & $15(16 \%)$ \\
Native aortic valve morphology & \\
Tricuspid & $46(51 \%)$ \\
Bicuspid & $38(42 \%)$ \\
Porcine valve dysfunction & $3(3 \%)$ \\
Homograft failure & $1(1 \%)$ \\
Mechanical prosthesis, root abscess & $1(1 \%)$ \\
Tetracuspid & $1(1 \%)$ \\
Unknown & $1(1 \%)$ \\
Hemodynamic lesion & \\
Aortic stenosis & \\
Aortic regurgitation & $45(50 \%)$ \\
Combined & $30(33 \%)$ \\
\hline
\end{tabular}

Values shown are means \pm standard deviations or numbers (percentages). NYHA, New York Heart Association.

digitally, and measurements were performed offline on a GE workstation (Echo PAC; GE Healthcare, Horten, Norway). Two experienced investigators performed the measurements.

\section{Measurements}

All measurements of dimensions were performed with calipers and according to the trailing edge to leading edge principle. The measurements in the aortic root and aorta were performed during systole. We measured the annulus from the insertion of the aortic cusp toward the interventricular septum and the anterior mitral leaflet, respectively. ${ }^{9}$ In control subjects, we measured the diameter of the sinus of Valsalva and the sinotubular junction. In patients with autografts, it was in most cases not possible to define any sinotubular junction (see Figures E1 and E2). Therefore only the sinus of Valsalva's diameter and the proximal part of the ascending aorta are reported. In the proximal ascending aorta or the ascending aorta from the right parasternal window (distal ascending aorta), we measured at the site with the widest diameter. In the aortic arch, we measured the diameter between the brachiocephalic trunk and the left common carotid artery (see Figure E1). The proximal descending aorta was measured after the left subclavian artery.

\section{Grading of Aortic Regurgitation}

The severity of aortic regurgitation was assessed by combining several parameters, as recommended by the American Society of Echocardiography. ${ }^{10}$ The variables included the width of the vena contracta with color Doppler scanning, the color Doppler area, the intensity of the continuous Doppler signal, and the degree of diastolic flow reversal in the proximal descending aorta. Aortic regurgitation was classified as mild, moderate, or severe.

\section{Statistical Analysis}

The dimensions of the aortic root and aorta are dependent on body surface area. From the healthy control subjects, regression equations 

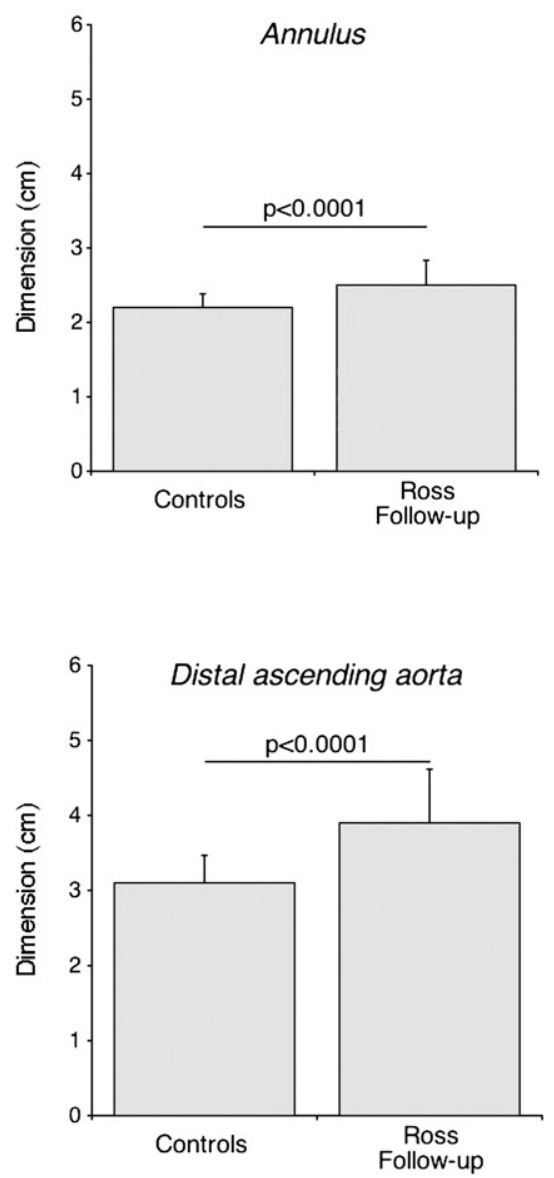

Sinus valsalva

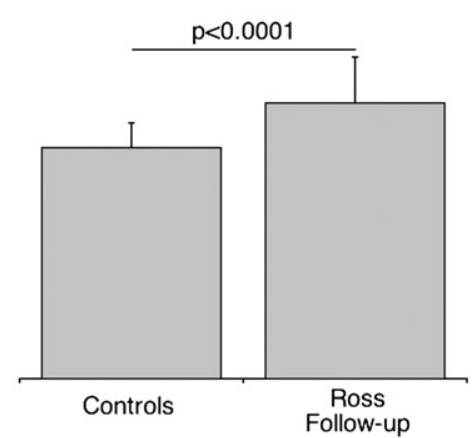

Aortic arch

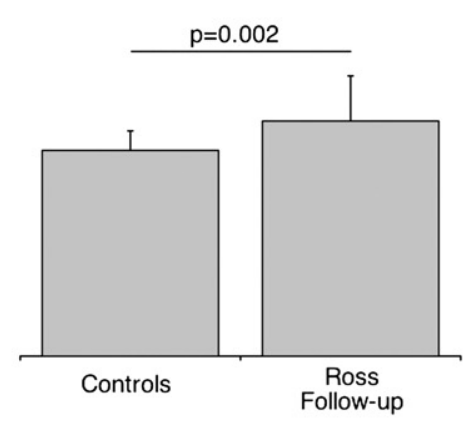

Proximal ascending aorta

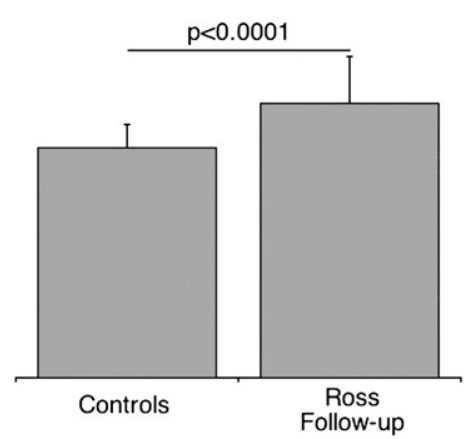

Proximal descending aorta

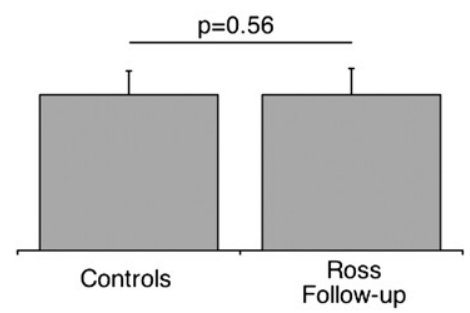

FIGURE 1. Aortic dimensions in patients undergoing the Ross procedure at the final follow-up and in age-matched control subjects.

were calculated, as well as residuals (see Table E1). For each patient in the study group, the expected dimensions were predicted. The observed values in patients were regarded as increased if they differed by more than 1.96 from the predicted value by $z$ score. When comparing paired data, a paired Student $t$ test (2-tailed) was used for continuous data, and the McNemar test was used for proportions. When comparing groups, the independentsamples $t$ test (2-tailed) was used to compare continuous data, and the Fisher exact test was used to compare proportions. For statistical analysis, IBM SPSS Statistics 17.0 software (SPSS, Inc, Chicago, Ill) was used. The present study is explorative, and therefore we did not perform any correction for multiplicity.

\section{RESULTS}

\section{Clinical Course}

Thirty-day mortality was $4(4.4 \%)$ of 91 . There were 9 late deaths: 1 bleeding complication after a second reoperation 9.4 years later, 2 myocardial infarctions (0.6 and 11.7 years after surgical intervention), 3 sudden deaths (4.4, 9.1, and 12.3 years postoperatively), and 3 noncardiac deaths.

Thirteen $(14 \%)$ patients underwent reoperations during follow-up. In addition, 5 of these patients underwent a second reoperation. Nine patients underwent reoperations because of autograft dysfunction. Five had dilatation of the autograft with significant secondary aortic regurgitation, 3 had cusp defects, and 1 had endocarditis. Reopera- tion on the homograft in the pulmonary artery position was performed in $3(3 \%)$ patients, and 1 patient underwent reoperation because of mitral regurgitation.

\section{Control Group}

The aortic dimensions in the control group and the regression equations with standard errors of the estimate are shown in Table E1. The linear relation between body surface area and dimensions was moderate to strong (correlation coefficients, 0.42-0.72). There was no significant difference in age $(54 \pm 12$ vs $51 \pm 9$ years, $P=.12)$ or body surface area $\left(1.9 \pm 0.3\right.$ vs $\left.1.8 \pm 0.2 \mathrm{~m}^{2}, P=.11\right)$ between patients undergoing the Ross procedure and control subjects at the end of follow-up. Figure E1 shows the aortic dimension measurements in the aortic root, the ascending aorta, and the aortic arch from the right parasternal position in a control subject.

\section{Autograft and Native Aortic Dilatation}

A comparison of autograft and native aortic dimensions between patients undergoing the Ross procedure and control subjects at the end of the follow-up period is shown in Figure 1. All parts of the aortic root and aorta, except 

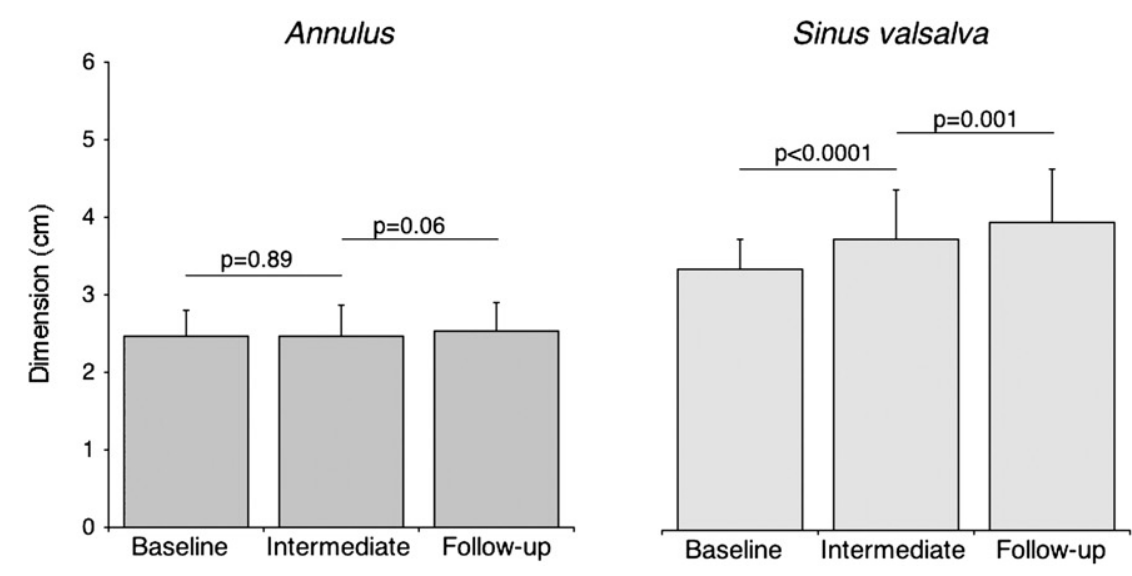

Proximal ascending aorta

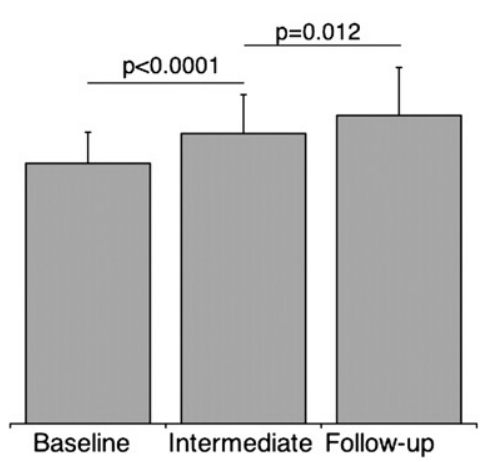

FIGURE 2. Aortic dimensions in patients undergoing the Ross procedure investigated on 3 occasions $(n=29)$.

the proximal part of the descending aorta, were significantly larger in patients compared with those in control subjects.

At the end of follow-up, 34 (48\%) of 71 patients had dimensions outside the expected normal range for the aortic root and aorta ( $z$ score $>1.96$ from the predicted value). Twenty $(28 \%)$ patients had enlargement in both the autograft and the native ascending aorta, $11(16 \%)$ patients had an isolated enlargement of the autograft, and $3(4 \%)$ patients had only enlargement of the native ascending aorta. The proportion of patients undergoing the Ross procedure with enlargement of the annulus was $39 \%$, with enlargement of the sinus of Valsalva was $30 \%$, with enlargement of the proximal ascending aorta was $43 \%$, with enlargement of the distal ascending aorta was $35 \%$, with enlargement of the aortic arch was $33 \%$, and with enlargement of the proximal descending aorta was $7 \%$. Six patients had aneurysmal dilatation $(>5 \mathrm{~cm})$ of the autograft, native aorta, or both.

There was no numeric difference between the proximal and distal ascending aortic diameter at the end of followup (mean difference, $0.0 \pm 0.53 \mathrm{~cm}$ ). However, in 26 $(40 \%)$ patients the native distal aorta was wider than the proximal part (Figure E2).

\section{Progression of Autograft and Native Aortic Dilatation}

The proportion of patients with an enlarged sinus of Valsalva of the autograft $(z$ score $>1.96)$ at the baseline investigation was $13 \%(8 / 63)$. This proportion increased to $33 \%$ $(21 / 63)$ at the end of follow-up $(P=.006)$. The sinus of Valsalva at baseline was $3.3 \pm 0.37 \mathrm{~cm}$, and at the end of follow-up, it was $3.7 \pm 0.65 \mathrm{~cm}(P<.0001)$. The proportion with an enlarged proximal ascending aorta was $16 \%(10 /$ $62)$ at baseline and $44 \%(27 / 62)$ at the end of follow-up $(P=.0014)$. The proximal ascending aorta at baseline was $3.3 \pm 0.39 \mathrm{~cm}$, and at the end of follow-up, it was 3.8 $\pm 0.68 \mathrm{~cm}(P<.0001)$. In the 29 patients with 3 complete postoperative echocardiographic investigations, the annular diameter did not change significantly between baseline and the intermediate follow-up investigation. The diameter of the sinus of Valsalva and the proximal part of the ascending aorta increased significantly from baseline to the intermediate investigation and continued to increase to the end of follow-up (Figure 2).

\section{Determinants of Aortic Dilatation}

Table 2 shows a comparison between patients with and without enlargement $(z$ score $>1.96)$ at the end of followup. Thirty-five patients had an enlarged aorta at followup. Enlargement of the aorta was not related to the bicuspid valve or postoperative hypertension. The pulmonary autograft dimension at the early baseline investigation was significantly larger in those who had enlargement at the second follow-up compared with those who did not.

\section{Effect of Reduction of Annular Size on Outcome}

Of the patients with complete echocardiographic followup, 54 of 71 underwent operations during a period during which aortic root reduction was performed if it was dilated compared with the pulmonary root. During this period,

TABLE 2. Determinants of aortic enlargement $(z$ score $>1.96)$ at the end of follow-up

\begin{tabular}{lccc}
\hline & $\begin{array}{c}\text { Not enlarged } \\
(\mathbf{n}=\mathbf{3 6})\end{array}$ & $\begin{array}{c}\text { Enlarged } \\
(\mathbf{n}=\mathbf{3 5})\end{array}$ & $\begin{array}{c}\boldsymbol{P} \\
\text { value }\end{array}$ \\
\hline Age at follow-up (y) & $55 \pm 12$ & $53 \pm 11$ & .39 \\
Female sex & $13(36 \%)$ & $9(26 \%)$ & .44 \\
Hypertension & $5(14 \%)$ & $6(17 \%)$ & .75 \\
Bicuspid & $16(44 \%)$ & $17(49 \%)$ & .81 \\
Preoperative AS & $22(61 \%)$ & $15(43 \%)$ & .16 \\
Preoperative AR & $12(33 \%)$ & $11(31 \%)$ & 1.00 \\
Baseline & & & \\
$\quad$ Annulus & $2.3 \pm 0.24$ & $2.4 \pm 0.3$ & .03 \\
$\quad$ Sinus of Valsalva & $3.1 \pm 0.37$ & $3.4 \pm 0.32$ & .003 \\
$\quad$ Proximal ascending aorta & $3.2 \pm 0.40$ & $3.5 \pm 0.34$ & .004 \\
\hline V
\end{tabular}

Values shown are means \pm standard deviations or numbers (percentages). $A S$, Aortic stenosis; $A R$, aortic regurgitation. 
aortic root reduction was performed in $31(57 \%)$ of 54 patients. Patients in the surgical correction group had larger annular and proximal ascending aortic dimensions compared with those in the uncorrected group at the preoperative investigation (Table 3). The surgical correction significantly reduced the annular diameter (from $2.6 \pm$ $0.36 \mathrm{~cm}$ preoperatively to $2.4 \pm 0.26 \mathrm{~cm}, P<.001$ ). At the baseline postoperative investigation, there was no difference in the sinus of Valsalva or proximal ascending aortic dimensions (Table 3), but a larger annulus was present in patients undergoing annular reduction. At the end of follow-up, there was no significant difference in autograft or native aortic dimensions between the surgically corrected and uncorrected groups.

\section{DISCUSSION}

The present comprehensive echocardiographic study with up to 14 years' follow-up reports for the first time that the dimensions of the native aorta increase in a significant number of patients over time after the Ross procedure in adults. In addition, a gradual dilatation of the autograft was reported.

Dilatation of the autograft is not a trivial complication after the Ross procedure. Severe dilatation of the autograft and native aorta with subsequent aortic regurgitation was a main reason for reoperation in our material ( $5 / 13$ patients undergoing reoperation). In our series, routine follow-up echocardiographic investigations showed enlargement of the autograft but in some cases also of the native aorta. This finding prompted us to perform a comprehensive transthoracic investigation of the aorta from the annulus to the proximal part of the descending aorta. This was obtained by adding a nonstandard projection where the patient lies in the right lateral position. The transducer is held close to the right parasternal border, and the beam direction is parallel with the parasternal border. Importantly, we found that the native distal part of the ascending aorta often was more dilated than the autograft. This finding underlines the importance of extending the investigation of patients after the Ross procedure to also include the native aorta.

There are many reports on autograft dilatation after the Ross procedure. ${ }^{2,411,12}$ However, the magnitude of the problem is controversial, and most reports do not include measurements of the native aorta. Dilatation of the native aorta has been discussed as a possible cause of autograft dilatation by Brown and associates, ${ }^{11}$ but the present study is, to our knowledge, the first that performs a comprehensive investigation of the native aorta.

The mechanism underlying the native aortic dilatation is unclear. The patients had no or only mild signs of atherosclerosis, and the group with enlargement at follow-up were not older than those who did not have enlargement. Dilatation and aneurysm formation can occur in association with genetic syndromes, such as Marfan syndrome.
TABLE 3. Patients from the second series $(n=54)$ : Patients surgically corrected $(n=31)$ because of right ventricular outflow tract/annular mismatch compared with uncorrected patients $(n=23)$

\begin{tabular}{|c|c|c|c|}
\hline & $\begin{array}{c}\text { Uncorrected } \\
\text { surgically }\end{array}$ & $\begin{array}{l}\text { Corrected } \\
\text { surgically }\end{array}$ & $\begin{array}{c}P \\
\text { value }\end{array}$ \\
\hline $\operatorname{BSA}\left(\mathrm{m}^{2}\right)$ & $1.91 \pm 0.32$ & $1.91 \pm 0.22$ & .88 \\
\hline \multicolumn{4}{|l|}{ Preoperative $(\mathrm{cm})$} \\
\hline Annulus & $2.4 \pm 0.19$ & $2.6 \pm 0.36$ & .011 \\
\hline Sinus of Valsalva & $3.4 \pm 0.6$ & $3.6 \pm 0.5$ & .23 \\
\hline Proximal ascending aorta & $3.4 \pm 0.75$ & $3.9 \pm 0.7$ & .03 \\
\hline \multicolumn{4}{|l|}{ Baseline $(\mathrm{cm})$} \\
\hline Annulus & $2.2 \pm 0.29$ & $2.4 \pm 0.26$ & .02 \\
\hline Sinus of Valsalva & $3.3 \pm 0.4$ & $3.3 \pm 0.4$ & .93 \\
\hline Proximal ascending aorta & $3.3 \pm 0.39$ & $3.3 \pm 0.43$ & .69 \\
\hline \multicolumn{4}{|l|}{ Follow-up (cm) } \\
\hline Annulus & $2.4 \pm 0.29$ & $2.5 \pm 0.33$ & .07 \\
\hline Sinus of Valsalva & $3.7 \pm 0.60$ & $3.6 \pm 0.62$ & .66 \\
\hline Proximal ascending aorta & $3.7 \pm 0.53$ & $3.7 \pm 0.66$ & .81 \\
\hline Distal ascending aorta & $3.7 \pm 0.61$ & $3.8 \pm 0.61$ & .65 \\
\hline Aortic arch & $3.1 \pm 0.53$ & $3.2 \pm 0.69$ & .68 \\
\hline Proximal descending aorta & $2.2 \pm 0.26$ & $2.1 \pm 0.44$ & .55 \\
\hline $\begin{array}{l}\text { Enlarged aorta }(z \text { score }>1.96) \\
\text { at the end of follow-up }\end{array}$ & $11(49 \%)$ & $17(55 \%)$ & .78 \\
\hline $\begin{array}{l}\text { Reoperations with dilatation at } \\
\text { the end of follow-up }\end{array}$ & $3(13 \%)$ & $1(3 \%)$ & .30 \\
\hline $\begin{array}{l}\text { Aortic regurgitation } \\
\text { ( } \geq \text { moderate)/reoperations at } \\
\text { the end of follow-up }\end{array}$ & $5(22 \%)$ & $9(29 \%)$ & .75 \\
\hline
\end{tabular}

A genetic analysis was not performed in our patients, but known Marfan syndrome is a contraindication for the Ross procedure at our institution. de Sa and colleagues ${ }^{13}$ have reported histologic evidence that patients with bicuspid aortic valve disease have more severe degenerative changes in the media of the ascending aorta and main pulmonary artery than patients with tricuspid aortic valve disease. However, whether patients with bicuspid valve disease should be candidates for the Ross procedure is controversial. $^{2,14}$ The present study includes a large proportion of patients with bicuspid aortic valve disease $(42 \%)$. The results of the present study do not support that bicuspid valve disease is a risk factor for dilatation of the autograft or native aorta because the dilatation was not more common in patients with bicuspid valves. In contrast, dilatation of the aorta at the end of follow-up was related to the baseline dimension of the autograft. The pulmonary autograft tissue is probably susceptible to dilate when exposed to the much higher systemic pressure, especially in those with a rather dilated and deformed aortic root at baseline. In 2 patients with the same blood pressure, according to the Laplace law, the wall tension will be highest in the patient with the widest aortic root. The pulmonary autograft does not have a sinotubular ridge, and this might further destabilize the root. The importance of the sinotubular ridge has been recognized by others, and David and coworkers ${ }^{2}$ 
have recommended stabilization with a circumferential piece of Dacron graft. Both the autograft and the native aorta were dilated at the end of follow-up, and the autograft and proximal part of the ascending aorta underwent progressive dilatation from baseline to the end of follow-up. One might speculate that the dilatation of the native aorta is secondary to the autograft dilatation.

Annular reduction, native aortic reduction, or both were performed in 31 of 54 patients in the second series, where mismatch between the aortic root and the pulmonary root was addressed. ${ }^{8}$ Patients in the surgically corrected group had significantly larger annular and proximal ascending aortic dimensions compared with those in the uncorrected patients at the preoperative investigation, and the surgical correction performed significantly reduced the annular diameter. At the end of follow-up, there was no significant difference in autograft or native aortic dimensions between the surgically corrected and uncorrected groups. If pre-existing pathology in the aortic wall expressed as preoperative dilatation was the cause of late dilatation, we could expect more dilatation at follow-up in the group with larger aortic roots preoperatively. Interestingly, our findings contrast those from a recent study by David and associates ${ }^{3}$ that reduction of the aortic annulus at the time of the operation did not resolve later problems.

In our series, 9 patients underwent reoperations because of autograft failure. Five patients had aortic regurgitation caused by root dilatation with inadequate cusp coaptation. In the remaining 4 patients, the underlying primary mechanism was cusp defects ( 1 endocarditis, 2 perforations of unclear cause, and 1 rupture). Cusp defects with regurgitation not related to autograft dilatation are not always well separated and defined in previous literature, ${ }^{1,11}$ and this might be another problem related to the high pressure and tension to the pulmonary tissue when exposed in the aortic position.

The long-term effect of aortic dilatation on the risk for reoperation is unclear, and only a close follow-up will definitely demonstrate whether native ascending aortic dilatation is a risk for reoperation after the Ross procedure.

Based on our results and our experience with the Ross procedure, our practice has been changed. Today, we are more restrictive and recommend this procedure to a more selected group of patients, such as women of childbearing age and young adult patients who require a high level of physical activity. Any candidate for the Ross procedure should receive comprehensive preoperative information, including the risk for reoperation.

\section{Study Limitations}

The comprehensive echocardiographic part of this study was limited to the intermediate and follow-up investigations. The distal part of the native aorta, including the aortic arch, was not studied preoperatively or at the first baseline investigation. Therefore it can be concluded that at the follow-up, the distal native aorta was dilated compared with that seen in control subjects, but the progression of the dilatation of the distal ascending aorta or the arch is unclear. Furthermore, it is difficult to distinguish between the autograft and the native aorta on the proximal ascending aorta measurements. Therefore the observed change in proximal ascending aortic diameter from baseline to follow-up conceivably reflects both autograft and native aortic enlargement.

Twelve patients were lost to follow-up. This was mainly due to logistic reasons: the patients were not willing to undertake a rather long journey to the hospital. However, we cannot exclude the possibility that these 12 patients had more pronounced dilatation of the aorta, and therefore our results might underestimate the problem.

In the present study, we used echocardiographic analysis to investigate the aorta, including the distal part of the ascending aorta, arch, and proximal part of the descending aorta. Echocardiographic analysis is not the best method to investigate these parts, and this might have introduced both overestimation and underestimation of the dimensions. Computed tomographic analysis or magnetic resonance imaging are better modalities. These methods were not used because of ethical (radiation) or availability reasons. We included a group with healthy control subjects, and this should compensate for some of the methodological shortcomings.

\section{Clinical Implications}

First, a close follow-up with echocardiographic investigations extended to include the native aorta is necessary to assess the dilatation of the pulmonary autograft and the native aorta after the Ross procedure. Second, patients with larger annular and proximal ascending aortic dimensions and mismatch between the aortic root and the pulmonary root can be surgically corrected and have the same autograft and native aortic dimensions compared with those seen in the uncorrected patients with no mismatch at late follow-up. Finally, in the present material patients with bicuspid valves did not have an increased risk for dilatation of the autograft and native aorta compared with patients with a tricuspid aortic valve.

We thank Lena Johansson, BSc, Department of Clinical Physiology, Sahlgrenska University Hospital, for her technical expertise in performing measurements. Sofia Zetterstrand, AstraZeneca, Mölndal, Sweden, provided statistical advice. The collaboration with hospitals in Western Sweden, which made this study possible, is greatly appreciated.

\section{References}

1. Chambers JC, Somerville J, Stone S, Ross DN. Pulmonary autograft procedure for aortic valve disease: long-term results of the pioneer series. Circulation 1997;96:2206-14. 
2. David TE, Omran A, Ivanov J, Armstrong S, de Sa MP, Sonnenberg B, et al. Dilation of the pulmonary autograft after the Ross procedure. J Thorac Cardiovasc Surg. 2000;119:210-20.

3. David TE, Woo A, Armstrong S, Maganti M. When is the Ross operation a good option to treat aortic valve disease? J Thorac Cardiovasc Surg. 2010;139:68-75.

4. Elkins RC, Thompson DM, Lane MM, Elkins CC, Peyton MD. Ross operation: 16-year experience. J Thorac Cardiovasc Surg. 2008;136:623-30. e1-5.

5. Luciani GB, Viscardi F, Pilati M, Prioli AM, Faggian G, Mazzucco A. The Ross-Yacoub procedure for aneurysmal autograft roots: a strategy to preserve autologous pulmonary valves. J Thorac Cardiovasc Surg. 2010;139:536-42.

6. Stelzer P, Jones DJ, Elkins RC. Aortic root replacement with pulmonary autograft. Circulation. 1989;80(suppl III):III209-13.

7. Ross DN. Replacement of aortic and mitral valves with a pulmonary autograft. Lancet. 1967;2:956-8.

8. Svensson G, Aljassim O, Svensson SE, Bech-Hanssen O, Kjellman U. Anatomical mismatch of the pulmonary autograft in the aortic root may be the cause of early aortic insufficiency after the Ross procedure. Eur J Cardiothorac Surg. 2002;21:1049-54.
9. Skjaerpe T, Hegranaes L, Hatle L. Noninvasive estimation of valve area in patients with aortic stenosis by Doppler ultrasound and two-dimensional echocardiography. Circulation. 1985;72:810-8.

10. Zoghbi WA, Enriquez-Sarano M, Foster E, Grayburn PA, Kraft CD, Levine RA, et al. Recommendations for evaluation of the severity of native valvular regurgitation with two-dimensional and Doppler echocardiography. J Am Soc Echocardiogr. 2003;16:777-802.

11. Brown JW, Ruzmetov M, Rodefeld MD, Mahomed Y, Turrentine MW. Incidence of and risk factors for pulmonary autograft dilation after Ross aortic valve replacement. Ann Thorac Surg. 2007;83:1781-9.

12. Hanke T, Stierle U, Boehm JO, Botha CA, Matthias Bechtel JF, Erasmi A, et al. Autograft regurgitation and aortic root dimensions after the Ross procedure: the German Ross Registry experience. Circulation. 2007;116(suppl I):I251-8.

13. de Sa M, Moshkovitz Y, Butany J, David TE. Histologic abnormalities of the ascending aorta and pulmonary trunk in patients with bicuspid aortic valve disease: clinical relevance to the Ross procedure. J Thorac Cardiovasc Surg. 1999;118:588-94.

14. Hanke T, Charitos EI, Stierle U, Robinson DR, Hemmer W, Moritz A, et al. The Ross operation-a feasible and safe option in the setting of a bicuspid aortic valve? Eur J Cardiothorac Surg. 2010;38:333-9. 

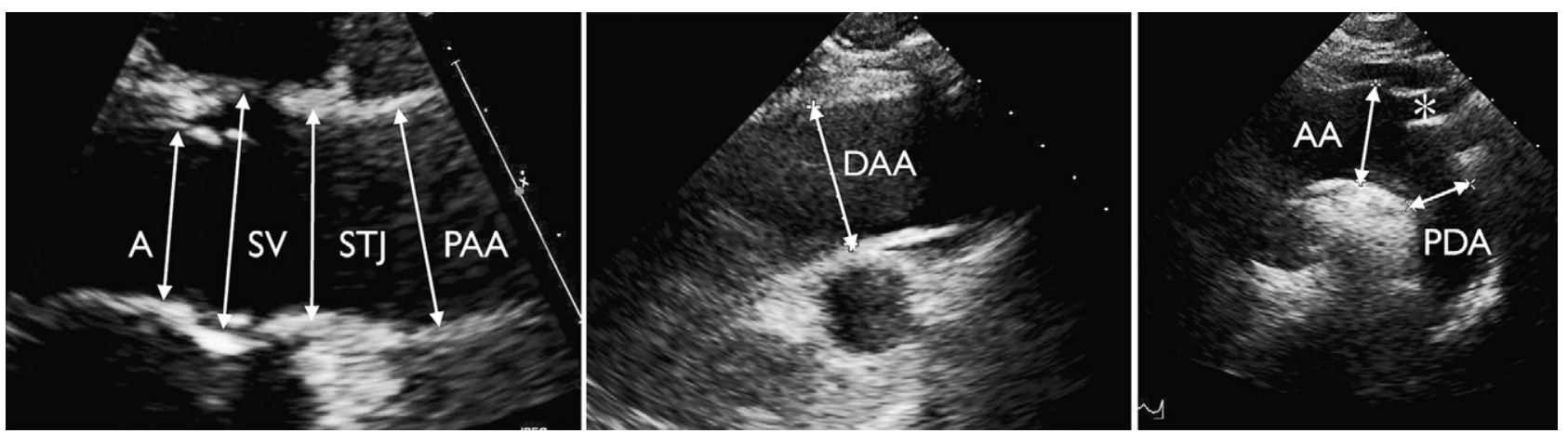

FIGURE E1. Parasternal long axis (left) of the aortic root in a control subject showing the annulus $(A ; 2.5 \mathrm{~cm})$, sinus of Valsalva $(S V ; 3.1 \mathrm{~cm})$, sinotubular junction $(S T J ; 2.7 \mathrm{~cm})$, and proximal ascending aorta $(P A A ; 3.0 \mathrm{~cm})$. To evaluate the middle and distal part of the ascending aorta, we investigated the patient in the right lateral position (middle). The long-axis projection through the aortic arch was obtained with the transducer in the suprasternal notch (right). $D A A$, Distal ascending aorta.The diameter $(A A ; 3.0 \mathrm{~cm})$ was measured proximal to the left common carotid artery $(*)$. The proximal part of the descending aorta $(P D A)$ was $2.1 \mathrm{~cm}$.
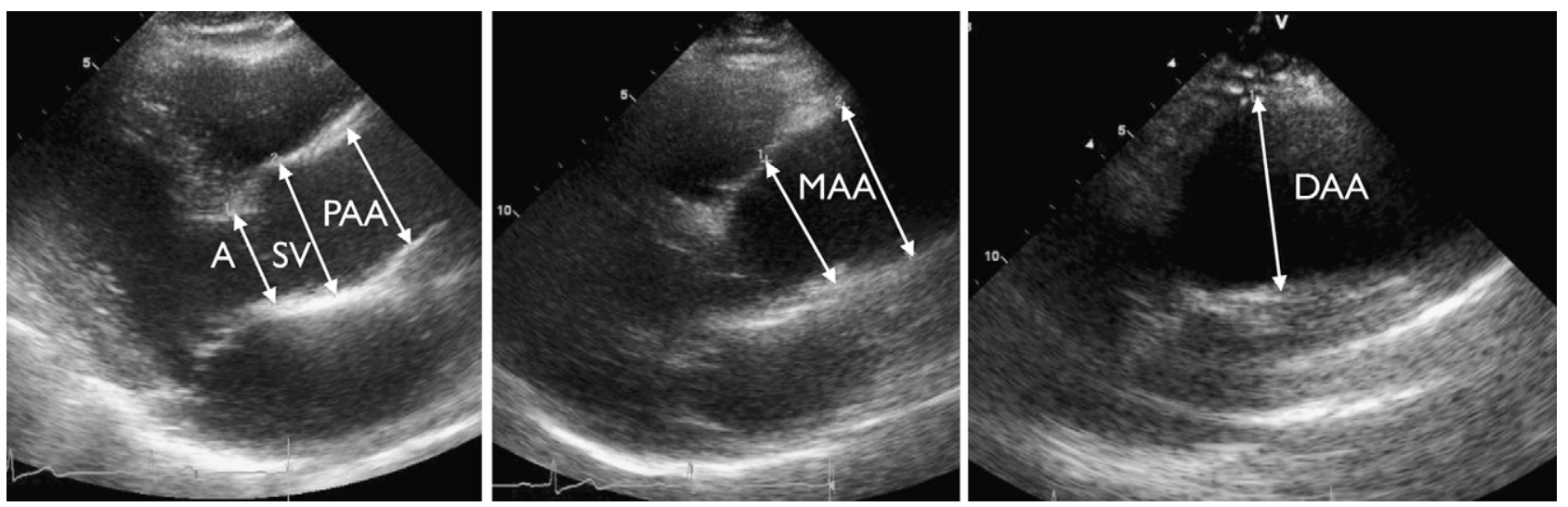

FIGURE E2. Parasternal long axis (left) of the autograft showing the annulus $(A ; 2.7 \mathrm{~cm})$, sinus of Valsalva $(S V ; 4.0 \mathrm{~cm})$, and proximal ascending aorta $(P A A ; 3.7 \mathrm{~cm})$. Note that there is no distinct sinotubular junction as in the control subject shown in Figure E1. Either the transducer was moved 1 intercostal space in the cranial direction (middle), the patient was investigated in the right lateral position (right), or both to investigate the native aorta. With this approach, the middle part of the native ascending aorta $(M A A)$ was $4.9 \mathrm{~cm}$, and the distal ascending aorta (DAA) was $5.1 \mathrm{~cm}$.

TABLE E1. Reference values for the annulus, sinus of Valsalva, sinotubular junction, ascending aorta, aortic arch, and proximal part of the descending aorta

\begin{tabular}{lcccr}
\hline \multicolumn{1}{c}{ Variable $(\mathbf{c m})$} & $\mathbf{n}$ & Mean \pm SD & Regression equation & Residual \\
\hline Annulus & 38 & $2.2 \pm 0.18$ & $0.82 \mathrm{BSA}+0.70$ & 0.13 \\
Sinus of Valsalva & 38 & $3.1 \pm 0.33$ & $1.2 \mathrm{BSA}+0.95$ & 0.72 \\
ST junction & 33 & $2.7 \pm 0.24$ & $0.94 \mathrm{BSA}+0.98$ & 0.28 \\
Proximal ascending aorta & 33 & $3.1 \pm 0.33$ & $1.25 \mathrm{BSA}+0.84$ & 0.18 \\
Distal ascending aorta & 28 & $3.1 \pm 0.37$ & $1.21 \mathrm{BSA}+0.93$ & 0.26 \\
Aortic arch & 26 & $2.8 \pm 0.27$ & $0.90 \mathrm{BSA}+1.1$ & 0.33 \\
Proximal descending aorta & 24 & $2.1 \pm 0.31$ & $0.93 \mathrm{BSA}+0.45$ & 0.62 \\
\hline
\end{tabular}

Mean body surface area \pm standard deviation was $1.83 \pm 0.16 \mathrm{~m}^{2}$ (range, $\left.1.60-2.15 \mathrm{~m}^{2}\right) . S D$, Standard deviation; $R$, correlation coefficient; BSA, body surface area; ST, sinotubular. 